AperTO - Archivio Istituzionale Open Access dell'Università di Torino

Differential diagnosis of pancreatic cysts: A prospective study on the role of intra-cystic glucose concentration

This is a pre print version of the following article:

Original Citation:

Availability:

This version is available http://hdl.handle.net/2318/1753205

since $2020-10-22 T 12: 48: 44 Z$

Published version:

DOI:10.1016/j.dld.2020.06.038

Terms of use:

Open Access

Anyone can freely access the full text of works made available as "Open Access". Works made available under a Creative Commons license can be used according to the terms and conditions of said license. Use of all other works requires consent of the right holder (author or publisher) if not exempted from copyright protection by the applicable law. 


\section{Differential diagnosis of pancreatic cysts: a prospective study on the role of intra-cystic glucose concentration}

Davide Giuseppe Ribaldone, $\mathrm{MD}^{1^{*}}$, Mauro Bruno, $\mathrm{MD}^{2 \star}$, Silvia Gaia, $\mathrm{MD}, \mathrm{PhD}^{2}$, Alessandro Cantamessa, $\mathrm{MD}^{2}$, Alberto Bragoni, $\mathrm{MD}^{3}$, Paola Caropreso, $\mathrm{MD}^{3}$, Marco Sacco, $\mathrm{MD}^{1,2}$, Sharmila Fagoonee, $\mathrm{PhD}^{4}$, Giorgio Maria Saracco, MD, Prof $^{1}$ and Claudio De Angelis, $\mathrm{MD}^{2}$

${ }^{1}$ Department of Medical Sciences, Division of Gastroenterology, University of Torino, Torino, Italy

${ }^{2}$ Department of General and Specialist Medicine, Gastroenterologia-U, Città della Salute e della Scienza di Torino, C.so Bramante 88, 10126 Turin, Italy ${ }^{3}$ Department of Laboratory Medicine, Città della Salute e della Scienza di Torino, C.so Bramante 88, 10126 Turin, Italy

${ }^{4}$ Institute of Biostructure and Bioimaging, CNR c/o Molecular Biotechnology Centre, 10126 Turin, Italy

${ }^{*}$ Co-first authors

Electronic word count (excluding abstract, references, tables, figures): $\underline{3991}$ 
Correspondence: Claudio De Angelis, Department of General and Specialist Medicine, Gastroenterologia-U, Città della Salute e della Scienza di Torino, C.so Bramante 88, 10126 Turin, Italy. Tel +390116335208 . Fax +39 0116336752, mail: eusdeang@hotmail.com;

Davide Giuseppe Ribaldone, Department of Medical Sciences, Division of Gastroenterology, University of Torino, Torino, Italy, C.so Bramante 88, 10126 Turin, Italy. Tel +39 0116333918. Fax +39 0116333623, mail: davrib_1998@yahoo.com

Conflicts of Interest: Claudio De Angelis is consultant for Boston Scientific Source of Funding: none declared. 


\section{Abstract}

Background: The accuracy and costs of current diagnostic methods in the differential diagnosis of pancreatic cystic lesions still has ample room for improvement.

Aims: The aim of the study was to confirm the diagnostic yield of intracystic glucose in the diagnosis of pancreatic cyst subtypes.

Methods: We prospectively recruited all patients who underwent Endoscopic Ultrasound with Fine Needle Aspiration of pancreatic cyst at our Institution.

Results: Fifty-six patients were included in the study. We found that intracystic glucose concentration $<50 \mathrm{mg} / \mathrm{dL}$ was significantly more sensitive than a concentration of Carcinoembryonic Antigen $>192 \mathrm{ng} / \mathrm{mL}(93.6 \%$ vs 54.8\%; $p=$ 0.003) for the diagnosis of mucinous cysts. In terms of specificity, the two markers were not different ( $96 \%$ vs $100 \% ; p=1)$. Regarding the diagnosis of nonmucinous cysts, intracystic glucose concentration $\geq 50 \mathrm{mg} / \mathrm{mL}$ showed higher sensitivity than Carcinoembryonic Antigen level $<5$ ng/mL (96\% vs 72\%) although a statistical significance could not be reached $(p=0.07)$. The two markers were not statistically different in terms of specificity (93.6\% vs $87.1 \% ; p$ $=0.62)$.

Conclusion: Given its diagnostic performance and ease of measurement, intracystic glucose may replace Carcinoembryonic Antigen in the differential diagnosis of mucinous versus non-mucinous pancreatic cysts.

Keywords: pancreatic cysts, intraductal papillary mucinous neoplasm; mucinous cystadenoma; serous cystadenoma, CEA, glucose 


\section{Introduction}

Pancreatic cystic lesions (PCLs) represent an increasingly important entity in clinical practice due to the fact that these are often found occasionally by noninvasive radiological examinations performed for other reasons. The prevalence of incidentally found pancreatic cysts in adulthood is high (between $2.6 \%$ and 19.6\%) [1,2]. PCLs are heterogeneous and include malignant, benign and premalignant lesions, capable of evolving into invasive carcinoma over time. Intraductal papillary mucinous neoplasm (IPMN), mucinous cystadenoma (MCA) and serous cystadenoma (SCA) are the three most frequent subtypes. Due to the increased prevalence of PCLs, also linked to the increase in the average age of the population [3], and their extensive heterogeneity of behaviour, it is imperative to accurately classify these lesions, in particular to distinguish benign forms from those with malignant potential, through the correct use of available resources and the development of new diagnostic tests.

The current guidelines [4-6] indicate that every patient with a new finding of pancreatic cysts should undergo a high-resolution radiological examination (if not yet performed), i.e. computed tomography (CT) with iodinated contrast medium or magnetic resonance with gadolinium (MR) associated with cholangiopancreatography (MRCP). These methods are used to obtain a correct differential diagnosis among the above-described entities and to identify any sign of malignant degeneration. However, their accuracy in differentiating benign cysts from potentially malignant cysts is limited and varies, depending on studies, between $20 \%$ and $80 \%[7,8]$. For the differential diagnosis of pancreatic cysts, it is therefore often necessary to resort to invasive imaging techniques. Not even 
Endoscopic Ultrasound (EUS) is accurate enough in the differential diagnosis of pancreatic cysts if one relies on morphological features only. This is particularly true for unilocular lesions in which the accuracy of EUS does not exceed $51 \%$ $[9,10]$.

Fine needle aspiration (FNA) may help in the differential diagnosis of PCLs but systematic reviews and metanalyses are concordant on the limited sensitivity of cytology obtained from pancreatic cysts $[11,12]$. Analysis of tumour markers in intra-cystic fluid has been extensively used to differentiate mucinous from nonmucinous cysts. Currently the most used marker is Carcinoembryonic Antigen (CEA). Several cut-offs $(30,192$ and $800 \mathrm{ng} / \mathrm{ml})$ have been proposed in the diagnosis of mucinous cysts. A concentration of $192 \mathrm{ng} / \mathrm{ml}$ is the most widely accepted because of an optimal "compromise" between sensitivity and specificity albeit a sensitivity as low as $51.9 \%$ has been reported in literature. For the diagnosis of non-mucinous lesions, a cut off of $5 \mathrm{ng} / \mathrm{ml}$ is usually employed [1013]. However, there is a large "gray area" in which the concentration of the intracystic CEA does not allow to ascertain the nature of the cyst.

Some studies [14-18] have analysed the role of intracystic glucose, with a cheaper and comparably accurate method, with respect to CEA in the differential diagnosis of PCLs. All of these underlined the need to validate the data with further studies. The aim of our study was to confirm the accuracy of intracystic glucose determination in differentiating mucinous from non-mucinous pancreatic cysts.

\section{Patients and Methods}


We performed a prospective, observational study in which all patients who underwent EUS-FNA of a pancreatic cyst at the Gastroenterology Unit of the University Hospital "Città della Salute e della Scienza di Torino" in Turin between October 2015 and September 2019 were enrolled.

Patients sent to our attention after detection of one or more pancreatic cysts, deemed worthy of further investigation, were selected. Our objective was to determine the nature of the cysts and establish a possible indication for surgical resection as well as the need for follow-up. All subjects had previously been investigated with at least one non-invasive II-level radiological examination such as CT or MR/MRCP which had not been exhaustive.

The EUS examinations were performed by three operators (M.B., C.D.A., S.G.) with broad experience in EUS (> 300/year). The procedures were carried out in deep sedation with anaesthesiological assistance using an Olympus GF-UCT 140 or 180 linear echoendoscope (Olympus Medical Systems, Tokyo, Japan). When deemed necessary by the endosonographer (evaluation of septa or mural nodules), an intravenous ultrasound contrast medium was administered (SonoVue ${ }^{\circledR}$, Bracco International BV, Netherlands) and a contrast-enhanced harmonic-EUS (CH-EUS) was performed. All the endoscopic procedures were digitally recorded.

FNAs were performed using an Expect $^{\circledR}$ Slimline 19-gauge flexible needle (Boston Scientific Corporation) or an EchoTip ${ }^{\circledR}$ Ultra 22-gauge needle (Cook Endoscopy). Fluid taken from each cyst (at least $1 \mathrm{ml}$ ) was sent for CEA, amylase and glucose determination and for cytological analysis. According to current literature, since intracystic Carbohydrate Antigen 19-9 (CA19-9) has a non- 
competitive diagnostic yield compared to CEA, we did not analyse its performance in this study [9].

In a subgroup of these patients, enrolled for another study carried out in our Unit, a needle-based confocal laser endomicroscopy ( $n C L E)$ was performed. The procedure was accomplished by preloading the AQ-Flex ${ }^{\circledR}$ confocal probe (Cellvizio $^{\circledR}$, Mauna Kea Technologies, Paris, France) in the 19-gauge needle. Once the needle was introduced into the cystic lesion, the probe was advanced beyond the tip of the needle by $1-2 \mathrm{~mm}$ and then placed in contact with the cyst wall. A fluorescent contrast agent was then intravenously administered $(2.5 \mathrm{ml}$ of $10 \%$ fluorescein sodium). After recording the images for a maximum of 4 minutes, the probe was extracted to allow aspiration of the intracystic fluid for the biochemical and cytological tests. The videos obtained were evaluated and interpreted "in vivo" by the operator and reviewed, if necessary, after storage.

The final diagnosis of cyst subtype was established on the basis of surgical pathology results (in case of subsequent surgical resection of the cyst), cytological examination of intracystic fluid (when conclusive) or nCLE (in case of no surgical procedure and inconclusive cytological examination).

In the subgroup of patients in whom none of the previous criteria could be met, the final diagnosis was made based on consensus among three experts (M.B., C.D.A., S.G.), blinded to cytological results, and on cyst morphology at CT, MR/MRCP, EUS and $\mathrm{CH}$-EUS.

A sub-analysis of patients whose diagnosis was made by surgical pathology results or conclusive cytological examination was performed. 


\section{Analysis of intracystic fluid}

A sample of aspirated liquid was sent to the Laboratory of our Hospital within 3 hours of the procedure. The concentration of CEA, amylase and glucose in the intracystic fluid was determined on a Cobas ${ }^{\circledR} 6000$ analyser (Roche Diagnostics). An immunometric technique was used for dosing CEA, whereas a spectrophotometric technique was employed for amylase and glucose analysis. Another sample of aspirated fluid was sent to the Pathology Department of our Hospital after inclusion in absolute alcohol. The cytological examination was performed using haematoxylin-eosin and Papanicolaou stainings [19].

From March 2018, to verify the feasibility of intracystic glucose, we decided that the cystic liquid samples were also analysed using a GEM ${ }^{\circledR}$ Premier $^{\circledR} 3000$ blood gas analysis instrument (Instrumentation Laboratory) with glucometers in use at our Hospital.

\section{Statistics}

The categorical variables were expressed as absolute numbers and percentages while continuous variables were expressed as mean \pm standard deviation (SD) or median and interquartile range (IQR) according to the distribution. The chisquare test was used for comparing categorical data.

To compare the average of two independent samples with normally distributed data, the t-test for independent samples was used. For non-normally distributed data (not even after logarithmic transformation), the non-parametric MannWhitney $U$ test was employed. The diagnostic performance of each test was evaluated by calculating sensitivity, specificity and accuracy. The diagnostic 
yields of the intracystic markers were compared using the McNemar test (according to the cut-off value available in literature $[14,15]$ ) and the ROC curve analysis.

The sample size of the study was calculated on the basis of the results of the study by Zikos et al. [15] (median glucose (interquartile range) 5 (5-17) mg/dl for mucinous cysts and 69 (17-102) mg/dl for non-mucinous cysts, mucinous/nonmucinous ratio 1.8/1, Type I error 0.05, Type II error 0.20): the minimum number of mucinous cysts was 8 , the minimum number of the non-mucinous cysts was 5 (minimum number of total of patients: 13). A $p$ value of less than 0.05 was considered significant.

Statistical analyses were performed using the Stata v 15 software (Stata Corp. LLC, College Station, Texas, USA) and R (version 3.3.1, R Project for Statistical Computing).

\section{Ethical considerations}

The demographic and clinical data as well as cyst features were prospectively collected in a database approved by the Ethical Committee. All patients signed an informed consent.

\section{Results}

During the study period (2015-2019), the total number of pancreatic cysts found by EUS-FNA in our institution was 516, and FNA was performed in 62 of these (12.0\%). Six cysts were excluded from the study: 2 due to presence of blood as 
this may alter cystic CEA and glucose determination, 3 due to presence of thick mucus or pus rendering the biochemical analysis difficult to perform, and 1 because the patient denied her consent.

Overall, 56 patients were included in the study (Figure 1): 36 were females (64.3\%), with a mean age of 62.6 years $(S D= \pm 14.4$ years).

Figure 1

The median of the largest diameter of pancreatic cysts revealed by EUS-FNA was $43 \mathrm{~mm}(\underline{I Q R}=26 \mathrm{~mm})$. Twenty-four (42.9\%) cysts were located in the pancreatic head, $27(48.2 \%)$ in the body, and $5(8.9 \%)$ in the tail. Twenty-eight cysts (50\%) were uniloculated and 28 (50\%) were multiloculated.

Twenty-five cysts were classified as non-mucinous (15 SCA, 8 PC and 2 cystic neuroendocrine tumors). Among the 31 mucinous cysts, 23 were IPMN, 7 MCA, and 1 cystadenocarcinoma. The definitive diagnosis was obtained, through histopathological examination of the surgical specimen, in 13 patients $(23.2 \%) \underline{7}$ mucinous cysts and 6 non-mucinous cysts).

Among the $43(76.8 \%)$ not-resected cysts, the final diagnosis was established in $11(19.6 \%)$ patients relying on cytological examination of the aspirated intracystic fluid, in 10 cases (17.9\%) on the basis of a typical nCLE pattern and in 22 cases (39.3\%) by consensus based on the judgment of three experts pancreatologists. 
In the group of mucinous cysts, patients were significantly older, and cysts were smaller, but no differences were found in gender and cysts' macroscopic appearance or location (Table 1).

Table 1

CEA

Intracystic CEA concentration was significantly higher in mucinous cysts than in non-mucinous cysts, with a median value of $247 \mathrm{ng} / \mathrm{mL}(\underline{\mathrm{QQR}}=1699 \mathrm{ng} / \mathrm{mL})$ in the former and of $1.6 \mathrm{ng} / \mathrm{mL}(\underline{I Q R}=6.9 \mathrm{ng} / \mathrm{mL})$ in the latter $(p<0.0001)$. A dot plot displaying CEA levels in mucinous and non-mucinous cysts is shown in Figure 2.

Figure 2

In identifying mucinous cysts, a concentration of CEA $>192 \mathrm{ng} / \mathrm{mL}$ demonstrated sensitivity, specificity and accuracy of $54.8 \%, 100 \%$ and $75 \%$, respectively.

On the other hand, CEA level $<5 \mathrm{ng} / \mathrm{mL}$ demonstrated sensitivity, specificity and accuracy of $72 \%, 87.1 \%$ and $80.4 \%$, respectively, in the diagnosis of nonmucinous cysts. 
In the ROC curve analysis, a concentration of CEA > $129 \mathrm{ng} / \mathrm{ml}$ was associated with a mucinous cyst with an $\mathrm{AUC}=0.94,95 \% \mathrm{IC}=0.84-0.98, p<0.0001$, sensitivity of $74.2 \%$ and specificity of $100 \%$.

\section{Glucose}

Intracystic glucose concentration was significantly lower in mucinous than in non-

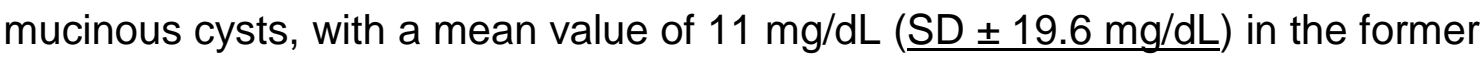

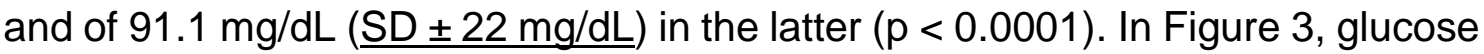
concentrations in mucinous and non-mucinous cysts are represented.

Figure 3

In the diagnosis of mucinous cysts, an intracystic glucose concentration $<50$ $\mathrm{mg} / \mathrm{dL}$ showed sensitivity, specificity and accuracy of $93.6 \%, 96 \%$ and $94.6 \%$, respectively. On the other hand, in the diagnosis of non-mucinous cysts, an intracystic glucose level of $\geq 50 \mathrm{mg} / \mathrm{dL}$ had sensitivity, specificity and accuracy of 96\%, 93.6\% and 94.6\%, respectively.

In the ROC curve analysis, a concentration of glucose $<49 \mathrm{mg} / \mathrm{dL}$ was associated with mucinous cyst with an $\mathrm{AUC}=0.98,95 \% \mathrm{IC}=0.91-1, p<0.0001$, sensitivity of $93.6 \%$ and specificity of $96 \%$.

As from March 2018, 18 EUS-FNA aspirates were analysed with two different blood gas analysers in use in our Unit and in the laboratory of our Hospital. Results were obtained with the blood gas analysers in 15 samples (in 3 samples, 
analysis could not be performed due to the large amount of mucus present). The discrepancy between the two results of the two analysers did not exceed $5 \mathrm{mg} / \mathrm{dL}$ meaning that no cysts would have been misclassified (mucinous vs nonmucinous) if the sample had been analysed only one of the two blood gas analysers.

In our population study, 3 patients had diabetes mellitus. However, blood glucose levels did not influence the intracystic glucose concentration: 2 subjects with IPMN (blood glucose level at 140 and $313 \mathrm{mg} / \mathrm{dl}$ on the day of the procedure) had intracystic glucose values of $3 \mathrm{mg} / \mathrm{dL}$ and $1 \mathrm{mg} / \mathrm{dL}$, respectively. One patient with SCA (blood glucose concentration of $130 \mathrm{mg} / \mathrm{dl}$ ) had an intracystic glucose concentration of $121 \mathrm{mg} / \mathrm{dL}$.

\section{Comparison between glucose and CEA}

In the diagnosis of mucinous cysts, intracystic glucose $<50 \mathrm{mg} / \mathrm{dL}$ was significantly more sensitive than a concentration of CEA $>192 \mathrm{ng} / \mathrm{mL}$ (sensitivity $93.6 \%$ vs $54.8 \% ; p=0.003)$. In terms of specificity, the two markers were not statistically different ( $96 \%$ vs $100 \%, p=1)$. On the other hand, in the diagnosis of non-mucinous cysts, an intracystic glucose concentration $\geq 50 \mathrm{mg} / \mathrm{mL}$ was more sensitive than a concentration of CEA $<5 \mathrm{ng} / \mathrm{mL}(96 \%$ vs $72 \%)$, but statistical significance was not reached $(p=0.07)$. In terms of specificity, the two markers were not statistically different $(93.6 \%$ vs $87.1 \%, p=0.62)$. A summary of the diagnostic yield of CEA and glucose is presented in Table 2.

Table 2 
When the ROC curve analyses of CEA and glucose were compared, no statistically significant difference was found $(p=0.12)$, but there was a tendency of superior accuracy for glucose (Figure 4).

Figure 4

The data on sensitivity, specificity and accuracy, considering only patients whose diagnosis was performed with surgical specimen (13) or cytological examination (11), are reported in Table 3.

Table 3

Association of tests: CEA or glucose

If an intracystic glucose value $<50 \mathrm{mg} / \mathrm{dL}$ or a value of CEA $>192 \mathrm{ng} / \mathrm{mL}$ in the diagnosis of mucinous cysts is considered, the values of sensitivity and accuracy slightly increased, compared to the sole glucose, respectively, to $96.8 \%$ and 96.4\% $(p=1)$ while specificity did not change $(96 \%)(p=1)$.

\section{Adverse events}

No clinical complications occurred during the procedures.

One patient (1.8\%) was admitted the day after the manoeuvre for mild acute pancreatitis that resolved uneventfully with medical therapy. 
No adverse events were recorded on a phone interview 30 days after the procedure.

\section{Discussion}

The major challenge, in the differential diagnosis of PCLs, is to accurately discriminate between asymptomatic benign cysts and malignant ones (or those with malignant potential). It is essential to avoid under-diagnosis of pancreatic cystic lesions with malignant potential in order to allow a proper follow-up or curative surgical therapy. Moreover, riskless benign cysts have to be correctly classified in order to avoid unnecessary surgery.

The discriminatory capacity of the currently available diagnostic tools is still considered inadequate by international guidelines [4-6] and, therefore, there is an urgent need for new methods or markers capable of differentiating the various subtypes of pancreatic cysts with greater precision.

Commercially available methods for CEA measurement have been validated for the analysis of serum or plasma but not for pancreatic cyst fluid, and there may be significant variability in the results among different methods and laboratories [20].

Unlike CEA, glucose measurement is undoubtedly a simple and easily available test in any laboratory, while the intracystic CEA assay is available only in reference centres. Moreover, it requires a lower volume of intracystic fluid than CEA assay (50 $\mu \mathrm{l}$ versus $500 \mu \mathrm{l})$ [15], and this can be particularly useful in clinical practice since sometimes only small amount of liquid can be drawn from a cyst. Furthermore, the spectrophotometric technique used for glucose determination 
is an extremely standardized and reproducible assay that does not present discrepancies among laboratories [17] and has a lower cost (1.26 $€$ vs $7.77 €$ for CEA in the present study).

The role of glucose concentration in intracystic fluid as a marker in the differential diagnosis of pancreatic cysts was first proposed by Park et al. in 2013 [16]. In the metabolomic analysis carried out on 45 pancreatic cysts that had undergone EUS-FNA, the intracystic glucose concentration was significantly higher in non-mucinous (SCA and PC) than in mucinous cysts (MCA and IPMN). In particular, a glucose concentration of less than $66 \mathrm{mg} / \mathrm{dL}$ showed sensitivity, specificity and accuracy of $95 \%, 56 \%$ and $84 \%$, respectively, in differentiating mucinous from non-mucinous cysts, and these values are comparable to those obtained with the intracystic CEA.

In 2015, a second prospective study [15] evaluated the performance of intracystic glucose level in the differential diagnosis of pancreatic cysts, using a lower concentration cut-off (50 mg/dL). The study, performed on 65 pancreatic cysts (42 mucinous and 23 non-mucinous), confirmed the statistically significant difference in intracystic glucose concentration between mucinous cysts (mean concentration $=5 \mathrm{mg} / \mathrm{dL}$ ) compared to non-mucinous ones (mean concentration $=69 \mathrm{mg} / \mathrm{dL})(p<0.0001)$. In the diagnosis of mucinous cysts, intracystic glucose concentration $<50 \mathrm{mg} / \mathrm{dL}$, determined by a laboratory spectrophotometric method, was more sensitive than intracystic CEA > $192 \mathrm{ng} / \mathrm{ml}$ (95\% vs 77\%, respectively). By using a combination of mucinous cysts glucose value $<50$ $\mathrm{mg} / \mathrm{dL}$ and a value of CEA $>192 \mathrm{ng} / \mathrm{mL}$, a sensitivity of $100 \%$ was obtained; however, the specificity was very low (33\%). 
A third prospective study conducted on 153 patients further strengthened these data, attributing even better diagnostic performance values to intracystic glucose value $<50 \mathrm{mg} / \mathrm{dL}$ (determined with simple glucometer) [14] with a sensitivity of $92 \%$, specificity of $87 \%$ and $90 \%$ accuracy in the diagnosis of mucinous cysts (95\%, 85\% and 93\%, respectively, when associated with the intracystic CEA > $192 \mathrm{ng} / \mathrm{mL})$.

A potential bias in these studies is that almost only cysts that underwent surgery (in the first study [16] 40/45 cysts, all cysts in the two other studies [14,15] so they cannot be considered representative of the vast majority of cysts found in daily practice) were included.

In a recent paper, Lopes et al. used intracystic CEA as the "gold standard" to compare intracystic glucose accuracy, and obtained an almost perfect correlation [17].

Finally, Faias and colleagues demonstrated, in a cohort of 82 frozen cystic fluids, that glucose concentration (dosed with a glucometer) outperformed CEA in sensitivity in the diagnosis of mucinous cysts [18].

Our study confirmed what emerged in previous studies by attributing an even better diagnostic performance to intracystic glucose concentration: in the diagnosis of mucinous cysts, a glucose value $<50 \mathrm{mg} / \mathrm{dL}$ showed sensitivity greater than $93 \%$ and specificity of $96 \%$, resulting in a correct classification of almost $95 \%$ of mucinous cysts. These parameters were better than those obtained with CEA > $192 \mathrm{ng} / \mathrm{mL}$ that demonstrated lower sensitivity (around 55\%) and was able to correctly classify "only" $75 \%$ of mucinous cysts. Similarly, an intracystic glucose value $>50 \mathrm{mg} / \mathrm{dL}$ identified non-mucinous cysts with greater 
sensitivity than CEA level $<5 \mathrm{ng} / \mathrm{mL}(96 \%$ vs $72 \%)$ albeit the statistical significance was not reached $(p=0.07)$, probably because of the relatively small sample size.

It is also interesting to note that upon combining an intracystic glucose value < $50 \mathrm{mg} / \mathrm{dL}$ or CEA value $>192 \mathrm{ng} / \mathrm{mL}$ (the positivity of at least one test is considered as sufficient) in the diagnosis of mucinous cysts, only a slight gain in sensitivity (96.8\%) and accuracy (96.4\%) $(p=1)$ was obtained compared to the tests considered individually, without any gain in terms of specificity (which remained at $96 \%)(p=1)$.

These data confirm the great value that the determination of intracystic glucose level can bring in clinical practice, allowing to discriminate with high reliability a non-mucinous cyst from a mucinous one.

This easy test can exceed the diagnostic performance of CEA hitherto considered sub-optimal [21] with considerable reduction in costs.

Furthermore, since none of the cysts would have been misclassified if the glucose determination had been accomplished with a simple blood gas analyser, a scenario could be imaginable in which the diagnosis of the type of cyst (mucinous vs non-mucinous), with excellent accuracy and very low costs, can be made immediately upon completion of the endoscopic procedure. Unlike two of the previous studies $[14,15]$, we did not use a portable glucometer because the model in use in our Unit is not suitable for biological fluids other than blood. The idea of using a blood gas analyser to dose intra-cystic glucose concentration came to us only in March 2018: we analysed 18 samples. In 15 samples the blood gas analyser gave results that were in line with those provided by the Cobas $^{\circledR} 6000$ 
Analyser. Unfortunately, in 3 samples, due to the mucus content, the sample could not be analysed with the blood gas analyser but only by Cobas $^{\circledR} 6000$ Analyser. So, we cannot state that the blood gas analyser can universally replace the more sophisticated analysis performed by a dedicated laboratory.

Some limitations of our study must be discussed. Intracystic glucose determination allows to discriminate between mucinous and non-mucinous cysts but, as CEA, cannot differentiate between IPMN and MCA or detect dysplasia or carcinoma. Similarly, regarding non-mucinous cysts, it cannot distinguish SCA from PC, solid pseudopapillary neoplasm (SPN) and cystic neuroendocrine tumours. This aspect represents the main limitation of any biochemical marker used in clinical practice but is not of secondary importance. Especially, in the group of non-mucinous cysts, the various subtypes have heterogeneous behaviour and different therapeutic indications (SCA, considered a benign form and devoid of surgical indications unless symptoms are present; cystic neuroendocrine tumor, for which for diameters $>2 \mathrm{~cm}$ surgical resection is always indicated; SPN in which surgical resection is always indicated [22]). A potential confounding factor, never reported in literature, not even in our study, could be represented by the infected cysts. It is well known that the presence of infection reduces the glucose content of effusions. Thus, albeit a rather rare event, this should be taken into consideration. A further critical issue regards the way in which the definitive diagnosis of the pancreatic cysts was obtained (i.e. the "diagnostic gold standard" against which the diagnostic yield of intracystic markers was calculated). In 32 patients (57.1\%), it was not possible to obtain a diagnosis of certainty based on a surgical specimen or cytology. In 10 subjects, 
a typical nCLE pattern can be considered a reliable surrogate according to current literature [23-25] but in 22 patients only a "consensus diagnosis" could be obtained. While, on one hand, this aspect represents a limitation of the study, on the other, it constitutes a strength in that it avoids an important selection bias present in most published studies. In fact, a sample consisting of only cysts that underwent surgical resection cannot be considered representative of all the cysts found in daily clinical practice, but only of those symptomatic or associated with "worrisome features". Furthermore, to further reduce the percentage of "uncertainty" in our study, we report herein that in 9 patients out of 22, the diagnosis can still be considered almost certain since in 5 patients, a communication between the cyst and the pancreatic duct was detected, and in 4 cases, there were multiple cysts, both aspects being highly suggestive of IPMN. Furthermore, we performed a sub-analysis on patients whose diagnosis was made by surgical pathology or cytological examination: the results did not change, rather the difference in the accuracy of glucose with respect to CEA increased, even if no statistical significance was reached due to the small sample size.

In conclusion, intracystic glucose is a rapid, inexpensive and easily performed test that can be performed in any laboratory and, according to our study, it has a better diagnostic performance compared to the most widespread and studied marker, the CEA. Given its simplicity and cost-effectiveness, we confirm that intracystic glucose determination should be introduced into clinical practice as an additional test in association with those already used, and in the future, it may supplant CEA. 


\section{References}

[1] Lee KS, Sekhar A, Rofsky NM, Pedrosa I. Prevalence of Incidental Pancreatic Cysts in the Adult Population on MR Imaging. Am J Gastroenterol 2010;105:2079-84. doi:10.1038/ajg.2010.122.

[2] Fernández-del Castillo C, Targarona J, Thayer SP, Rattner DW, Brugge WR, Warshaw AL. Incidental pancreatic cysts: clinicopathologic characteristics and comparison with symptomatic patients. Arch Surg 2003;138:427-3; discussion 433-4. doi:10.1001/archsurg.138.4.427.

[3] Kimura W, Nagai H, Kuroda A, Muto T, Esaki Y. Analysis of small cystic lesions of the pancreas. Int J Pancreatol 1995;18:197-206. doi:10.1007/BF02784942.

[4] Buscarini E, Pezzilli R, Cannizzaro R, De Angelis C, Gion M, Morana G, et al. Italian consensus guidelines for the diagnostic work-up and followup of cystic pancreatic neoplasms. Dig Liver Dis 2014;46:479-93. doi:10.1016/j.dld.2013.12.019.

[5] European Study Group on Cystic Tumours of the Pancreas. European evidence-based guidelines on pancreatic cystic neoplasms. Gut 2018;67:789-804. doi:10.1136/gutjnl-2018-316027.

[6] Elta GH, Enestvedt BK, Sauer BG, Lennon AM. ACG Clinical Guideline: Diagnosis and Management of Pancreatic Cysts. Am J Gastroenterol 2018;113:464-79. doi:10.1038/ajg.2018.14.

[7] Kehagias D, Smyrniotis V, Kalovidouris A, Gouliamos A, Kostopanagiotou 
E, Vassiliou J, et al. Cystic tumors of the pancreas: preoperative imaging, diagnosis, and treatment. Int Surg n.d.;87:171-4.

[8] Visser BC, Yeh BM, Qayyum A, Way LW, McCulloch CE, Coakley F V. Characterization of Cystic Pancreatic Masses: Relative Accuracy of CT and MRI. Am J Roentgenol 2007;189:648-56. doi:10.2214/AJR.07.2365.

[9] Oh H-C, Kim M-H, Hwang CY, Lee TY, Lee SS, Seo D-W, et al. Cystic lesions of the pancreas: challenging issues in clinical practice. Am J Gastroenterol 2008;103:229-39; quiz 228, 240. doi:10.1111/j.15720241.2007.01558.x.

[10] Brugge WR, Lewandrowski K, Lee-Lewandrowski E, Centeno BA, Szydlo T, Regan S, et al. Diagnosis of pancreatic cystic neoplasms: a report of the cooperative pancreatic cyst study. Gastroenterology 2004;126:13306. doi:10.1053/j.gastro.2004.02.013.

[11] de Jong K, Poley J-W, van Hooft JE, Visser M, Bruno MJ, Fockens P. Endoscopic ultrasound-guided fine-needle aspiration of pancreatic cystic lesions provides inadequate material for cytology and laboratory analysis: initial results from a prospective study. Endoscopy 2011;43:585-90. doi:10.1055/s-0030-1256440.

[12] Thosani N, Thosani S, Qiao W, Fleming JB, Bhutani MS, Guha S. Role of EUS-FNA-based cytology in the diagnosis of mucinous pancreatic cystic lesions: a systematic review and meta-analysis. Dig Dis Sci 2010;55:2756-66. doi:10.1007/s10620-010-1361-8.

[13] Park WG-U, Mascarenhas R, Palaez-Luna M, Smyrk TC, O’Kane D, Clain JE, et al. Diagnostic Performance of Cyst Fluid Carcinoembryonic Antigen 
and Amylase in Histologically Confirmed Pancreatic Cysts. Pancreas 2011;40:42-5. doi:10.1097/MPA.0b013e3181f69f36.

[14] Carr RA, Yip-Schneider MT, Simpson RE, Dolejs S, Schneider JG, Wu H, et al. Pancreatic cyst fluid glucose: rapid, inexpensive, and accurate diagnosis of mucinous pancreatic cysts. Surgery 2018;163:600-5. doi:10.1016/j.surg.2017.09.051.

[15] Zikos T, Pham K, Bowen R, Chen AM, Banerjee S, Friedland S, et al. Cyst Fluid Glucose is Rapidly Feasible and Accurate in Diagnosing Mucinous Pancreatic Cysts. Am J Gastroenterol 2015;110:909-14. doi:10.1038/ajg.2015.148.

[16] Park WG, Wu M, Bowen R, Zheng M, Fitch WL, Pai RK, et al. Metabolomic-derived novel cyst fluid biomarkers for pancreatic cysts: glucose and kynurenine. Gastrointest Endosc 2013;78:295-302.e2. doi:10.1016/j.gie.2013.02.037.

[17] Lopes CV. Cyst fluid glucose: An alternative to carcinoembryonic antigen for pancreatic mucinous cysts. World J Gastroenterol 2019;25:2271-8. doi:10.3748/wjg.v25.i19.2271.

[18] Faias S, Pereira L, Roque R, Chaves P, Torres J, Cravo M, et al. Excellent Accuracy of Glucose Level in Cystic Fluid for Diagnosis of Pancreatic Mucinous Cysts. Dig Dis Sci 2019. doi:10.1007/s10620-01905936-5.

[19] Pitman M, Centeno B, Genevay M, Stelow E, Mino-Kenudson M, Schmidt $\mathrm{CM}$, et al. Standardized terminology and nomenclature for pancreatobiliary cytology: The Papanicolaou Society of Cytopathology 
Guidelines. Cytojournal 2014;11:15. doi:10.4103/1742-6413.133343.

[20] Boot C. A review of pancreatic cyst fluid analysis in the differential diagnosis of pancreatic cyst lesions. Ann Clin Biochem 2014;51:151-66. doi:10.1177/0004563213503819.

[21] Gaddam S, Ge PS, Keach JW, Mullady D, Fukami N, Edmundowicz SA, et al. Suboptimal accuracy of carcinoembryonic antigen in differentiation of mucinous and nonmucinous pancreatic cysts: results of a large multicenter study. Gastrointest Endosc 2015;82:1060-9. doi:10.1016/j.gie.2015.04.040.

[22] Scholten L, van Huijgevoort NCM, van Hooft JE, Besselink MG, Del Chiaro M. Pancreatic Cystic Neoplasms: Different Types, Different Management, New Guidelines. Visc Med 2018;34:173-7. doi:10.1159/000489641.

[23] Konda VJA, Meining A, Jamil LH, Giovannini M, Hwang JH, Wallace MB, et al. A pilot study of in vivo identification of pancreatic cystic neoplasms with needle-based confocal laser endomicroscopy under endosonographic guidance. Endoscopy 2013;45:1006-13. doi:10.1055/s0033-1344714.

[24] Nakai Y, Iwashita T, Park DH, Samarasena JB, Lee JG, Chang KJ. Diagnosis of pancreatic cysts: EUS-guided, through-the-needle confocal laser-induced endomicroscopy and cystoscopy trial: DETECT study. Gastrointest Endosc 2015;81:1204-14. doi:10.1016/j.gie.2014.10.025.

[25] Napoléon B, Lemaistre AI, Pujol B, Caillol F, Lucidarme D, Bourdariat R, et al. A novel approach to the diagnosis of pancreatic serous 
cystadenoma: Needle-based confocal laser endomicroscopy. Endoscopy 1998;47:26-32. doi:10.1055/s-0034-1390693. 
Figure 1. Flow chart of patients.

Figure 2. Dot plot of CEA concentration in mucinous and non-mucinous cysts. Dashed lines identify the cut-off values of 5 and $192 \mathrm{ng} / \mathrm{mL}$. The continuous horizontal lines represent median values. The y-axis is plotted on a logarithmic scale.

Figure 3. Dot plot of glucose concentration in mucinous and non-mucinous cysts. Dashed line identifies the cut-off value of $50 \mathrm{mg} / \mathrm{dL}$. The continuous horizontal lines represent mean values.

Figure 4. Comparison of ROC curve analyses of CEA and glucose. 